\title{
AN ELASTIC MEMS DEVICE FOR CELLULAR FORCE MEASUREMENT
}

\author{
Yu Sun, Xinyu Liu, Wenhui Wang, Bob M. Lansdorp \\ Advanced Micro and Nanosystems Laboratory, University of Toronto \\ Toronto, Ontario, Canada
}

\begin{abstract}
This paper presents a PDMS (polydimethylsiloxane) cell holding device and a sub-pixel visual tracking algorithm that are used together to visually resolve applied forces to a single cell at a full $30 \mathrm{~Hz}$ with a sub- $\mu \mathrm{N}$ resolution. The computer vision microscopy based measurement technique and a further scaleddown device will provide a $0.52 \mathrm{nN}$ resolution, resulting in a novel platform for investigating the mechanical properties of many cell types of different sizes and for providing real-time cellular force feedback during automated microrobotic cell manipulation.
\end{abstract}

\section{INTRODUCTION}

Mechanical force plays a critical role in the interactions of biological cells with their surrounding environment. In order to understand how mechanical interactions affect the function of biological cells, cellular forces must be quantitated and their effects on the function and morphology of cells be studied. The capability of precisely measuring small forces is also important for providing force feedback during microrobotic biomanipulation where biological cells being handled are easily damaged.

Compared to cellular force measurement techniques such as optical tweezers, atomic force microscopy, magnetic beads, and micropipette aspiration, MEMS force sensors [1][2] are costeffective and provide flexibility for system integration. However, the silicon-based devices require sophisticated equipment sets and much processing effort. Moreover, issues such as biocompatibility and operating in an aqueous environment for biological cells to survive often pose stringent challenges and intricacies in MEMS design, material selection, and microfabrication.

As microrobotic biomanipulation and single cell mechanical characterization in cellular biomechanics are always conducted under an optical microscope, it is highly desirable to simultaneously obtain high-resolution force measurements extracted from microscopy vision feedback. Thus, no distinct sensing modalities are required and a single vision sensor (CCD/CMOS camera) is capable of providing two forms of feedback (i.e., vision and force). For cellular force sensing, this concept can be implemented by visually tracking deflections of elastic, low-stiffness microstructures, and subsequently, transforming material deflections into forces.

Recently, microfabricated elastomer post/pillar structures using PDMS were constructed to visually measure traction forces generated by biological cells (smooth muscle cells [3] and cardiac myocytes [4]). PDMS-based devices are biocompatible and can be readily constructed using soft lithography without requiring intensive microfabrication efforts or sophisticated equipment sets. In [3][4], off-line image post-processing was conducted to measure PDMS post deflections. The cellular force measurement accuracy in these studies was not warranted because the smalldeflection assumption in linear elasticity was not satisfied and the shear component of deflected posts not considered. Additionally, without adopting sub-pixel visual feature tracking techniques, the cellular force measurement resolution was sacrificed.

Although the PDMS devices [3][4] can be applied to measuring how much forces are generated by adherent cells at adhesion sites on top of posts, they do not allow for studying how externally applied forces affect cell responses, which is particularly true for suspended cells. A range of applications call

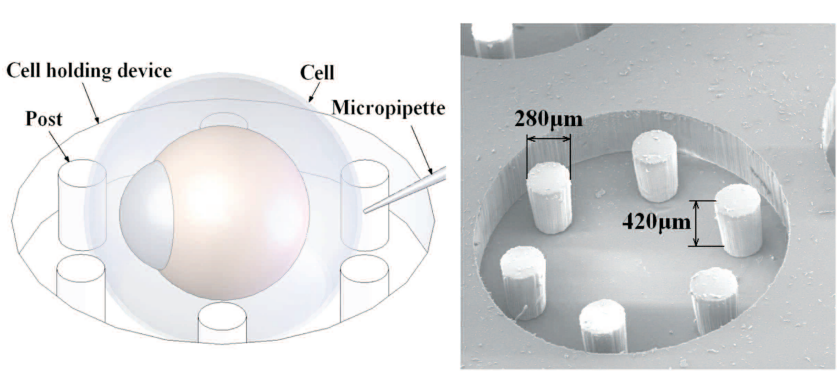

Figure 1. SEM image of a PDMS cell holding device.

for a new experimental platform including force-controlled microrobotic cell injection (e.g., injecting genetic materials into individual cells to treat severe infertility or to create transgenic organisms for cancer studies [5]) and cell indentation for mechanical property characterization in which forces applied by a micropipette or indenter to a cell must be measured in real time.

\section{EXPERIMENTAL SETUP}

The system consists of a PDMS cell holding device, an optical microscope with a CMOS camera, and a three-degrees-offreedom microrobot with a $40 \mathrm{~nm}$ positioning resolution along each axis. The microrobot controls the motion of an injection micropipette to apply forces to a single biological cell that is surrounded by low-stiffness protruding posts inside a cavity. Although a single element is shown in Figure 1, there are arrays of such elements on the PDMS cell holding device. The system setup is mounted on a vibration isolation table for minimizing vibrationinduced vision/force measurement errors.

The PDMS cell holding device was constructed using standard soft lithography with SU-8 2075 (MicroChem, U.S.) and a ratio of 10:1 for Sylgard 184 (Dow Corning, U.S.) and curing agent. After a molding process, the PDMS cell holding device was peeled off the SU-8 template. The depth of the cavity and protruding posts is $420 \mu \mathrm{m}$, and the diameter of the posts is $280 \mu \mathrm{m}$. The devices were plasma treated before use.

\section{MATERIALS AND METHODS}

The zebrafish has emerged as an important model organism for development and genetic studies. The large size of zebrafish embryos ( $\sim 1.2 \mathrm{~mm}$ including chorion that is the outside membrane in Figure 2) makes the handling and manipulation of these cells less demanding. Although zebrafish embryos were chosen in this study to demonstrate vision-based cellular force sensing, the PDMS cell holding device can be readily scaled down for studying cells of smaller sizes (e.g., mouse embryos) and the methodology is not scale or cell type dependent.

Figure 2 shows an image captured in the cell indentation experiments. An injection micropipette mounted on the microrobot is employed to exert an indentation force to a zebrafish embryo, which deflects the two supporting posts against the cell. Post deflections, measured in real time by a visual tracking algorithm, are fitted to an analytical mechanics model to obtain the injection/indentation force ( $F$ in Figure 2). A valid application of linear elasticity was guaranteed as the maximum value of the measured deflection slope of the posts' free ends is $13.7^{\circ}$, which satisfies $\sin \theta \approx \theta$ and thus, the small deflection assumption. 
Because the ratio of post height $(420 \mu \mathrm{m})$ to diameter $(280 \mu \mathrm{m})$ in this design does not satisfy the pure bending assumption of linear elastic beams (i.e., height to diameter ratio must be greater than 5) [6], both bending and shearing must be considered to accurately map the deflection to the applied force

$$
F=\sum_{i=1}^{2} \frac{\delta_{h i}}{\frac{64 H^{3}}{3 \pi E D^{4}}+\frac{20(1+\gamma) H}{9 A E}}
$$

where $i=1,2 ; \delta_{h i}$ is horizontal deflection; $H$ and $A$ are post height and cross sectional area; $E$ and $\gamma$ are Young's modulus and Poisson's ratio $(\gamma=0.5$ for PDMS). It is known that different processing conditions of PDMS result in large variations of Young's modulus values. In order to accurately determine the Young's modulus value, calibration experiments were conducted with a piezoresistive silicon force sensor (AE801, SensorOne, U.S.) on a PDMS cantilever beam produced under the same processing conditions. The Young's modulus value was determined to be $707.2 \mathrm{kPa}$.

A sub-pixel visual tracking algorithm was developed to accurately track post deflections in real time. The task is two fold, to track image patches of the top surfaces of supporting posts as well as to accurately detect the circular center positions. A template matching algorithm with template update was used to track the motion of the supporting posts. After tracking the image patches that contain post top surfaces, the circular center positions were detected by a least squares circle detection (LSCD) algorithm. Accumulative errors caused by template updating were eliminated in the subsequent LSCD detection of circular centers.

\section{RESULTS AND DISCUSSION}

A $2.5 \times$ objective (NA 0.07 ) was first used in order to have both the cell and supporting posts inside the field of view. The calibrated pixel size is $4.17 \mu \mathrm{m}$. The micropipette used for indenting cells has an outside diameter of $25 \mu \mathrm{m}$ at the tip end. The indentation speed was controlled at $600 \mu \mathrm{m} / \mathrm{sec}$. The sub-pixel visual tracking algorithm has a tracking resolution of 0.45 pixel and costs $22.3 \mathrm{~ms}$ for processing each frame of image, proving the real-time capability $(30 \mathrm{~Hz})$. Measured horizontal deflections were substituted into the mechanics model (1) to calculate indentation forces. In Figure 3, cell deformations on the micropipette side

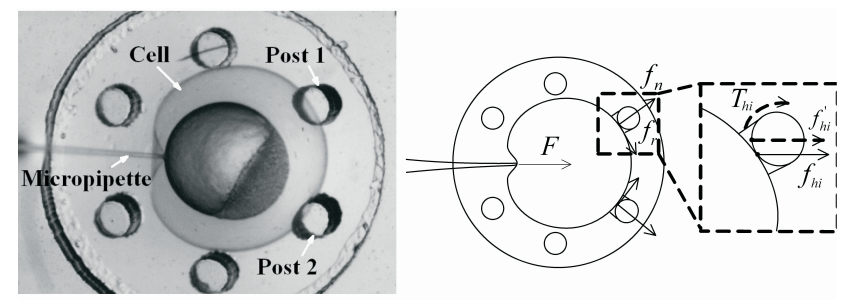

Figure 2. Indentation forces cause two supporting posts to deflect (left) and force analysis (right).

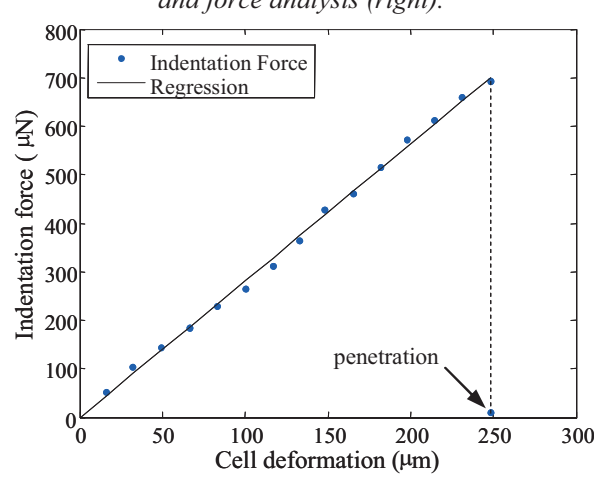

Figure 3. Force-deformation curve of an indented zebrafish embryo.
TABLE I. Vision-BASED CELlular ForCE MEASUREMENT RESOLUTION.

\begin{tabular}{|c|c|c|c|c|c|}
\hline $\begin{array}{c}\text { Objective } \\
\text { magnification }\end{array}$ & NA & $\begin{array}{c}\text { Rayleigh } \\
\text { limit } \\
(\mu \mathrm{m})\end{array}$ & $\begin{array}{c}\text { Pixel } \\
\text { size } \\
(\mu \mathrm{m})\end{array}$ & $\begin{array}{c}\text { Deflection } \\
\text { resolution } \\
(\text { pixel })\end{array}$ & $\begin{array}{c}\text { Force } \\
\text { resolution } \\
(\mu \mathrm{N})\end{array}$ \\
\hline $2.5 \times$ & 0.07 & 3.93 & 4.17 & 0.45 & 12.7 \\
\hline $4 \times$ & 0.13 & 2.12 & 2.27 & 0.58 & 8.9 \\
\hline $6.4 \times$ & 0.13 & 2.12 & 1.56 & 0.40 & 4.2 \\
\hline $10 \times$ & 0.30 & 0.92 & 1.00 & 0.54 & 3.7 \\
\hline
\end{tabular}

were obtained by subtracting the average horizontal deflections of the two posts from the micropipette's displacement (i.e., displacement of the microrobot). With the $2.5 \times$ objective, the vision-based force measurement resolution is $12.7 \mu \mathrm{N}$. The force measurement resolution depends on optical conditions and is greatly improved with objectives of higher magnifications. As shown in Table I, with a $10 \times$ objective (NA 0.3), the current system is capable of visually resolving forces down to $3.7 \mu \mathrm{N}$.

Besides the applicability to cellular biomechanics, the device and tracking algorithm can provide important force feedback in microrobotic cell injection. Cell membrane penetration can be precisely determined from the vision-based force measurement results for subsequent genetic material deposition (Figure 3). The abrupt position change of the supporting posts signals cell membrane penetration. Forces required to penetrate the zebrafish chorion were determined to be $692.8 \mu \mathrm{N}$, in agreement with previously reported results [7].

Importantly, the vision-based cellular force sensing framework is not scale dependent. A cell holding device with supporting posts of $25 \mu \mathrm{m}$ in height and $5 \mu \mathrm{m}$ in diameter, based on a 0.5 pixel visual tracking resolution obtained in this study, has the capability of visually resolving forces down to $0.52 \mathrm{nN}$ with a $40 \times$ objective, providing an extremely cost-effective, yet powerful experimental platform for single cell studies with a sub- $\mathrm{nN}$ force measurement resolution.

\section{CONCLUSION}

This paper presented a method to robustly measure forces applied to single biological cells through the use of a microfabricated PDMS device and a sub-pixel computer vision tracking algorithm. The current system is capable of resolving cellular forces down to $3.7 \mu \mathrm{N}$. A realistic estimate reveals that the methodology presented in this paper is capable of resolving forces down to $0.52 \mathrm{nN}$ with a scaled-down device (supporting posts of $25 \mu \mathrm{m}$ in height and $5 \mu \mathrm{m}$ in diameter). The device design and experimental technique are applicable to investigating mechanical properties of many cell types of different sizes and to providing real-time force feedback during microrobotic cell manipulation.

\section{REFERENCES}

[1] Y. Sun, K.T. Wan, B.J. Nelson, J. Bischof, and K. Roberts, "Mechanical property characterization of the mouse zona pellucida," IEEE Trans. on NanoBioScience, 279, 2 (2003).

[2] G. Lin, K.S. Pister, and K.P. Roos, "Surface micromachined polysilicon heart cell force transducer,' J. of MEMS, 9, 9 (2000).

[3] J.L. Tan, J. Tien, D.M. Pirone, D.S. Gary, K. Bhadriraju, C.S. Chen, "Cells lying on a bed of microneedles: an approach to isolate mechanical force," Proc. Natl. Acad. Sci., 1484, 100 (2003).

[4] Y. Zhao and X. Zhang, "Cellular mechanics study in cardiac myocytes using PDMS pillars array," Sensors and Actuators A, 398, 125 (2006)

[5] Y. Sun and B.J. Nelson, "Biological cell injection using an autonomous microrobotic system," International J. of Robotics Research, 861, 21 (2002).

[6] A.C. Ugural and S.K. Fenster, Advanced Strength and Applied Elasticity, Prentice Hall, 2003.

[7] D.H. Kim, Y. Sun, S. Yun, S.H. Lee, and B.K. Kim, "Investigating chorion softening of zebrafish embryos with a microrobotic force sensing system," J. of Biomechanics, 1359, 38 (2005). 